\section{Comments on "Fuzzy Control Systems Design via Fuzzy Lyapunov Functions"}

K. Guelton, Member, IEEE, T.M. Guerra, M. Bernal, T. Bouarar and N. Manamanni, Member, IEEE

\begin{abstract}
This note considers the work untitled "Fuzzy Control Systems Design via Fuzzy Lyapunov Functions" published by J. Li, S. Zhou and S. Xu in IEEE Trans. on SMC part $B$ [1]. In the latter, the authors try to extend the work of Rhee and Won [2]. Nevertheless, the results proposed in [1] have been obtained without taking into account some necessary conditions to ensure the line integral function to be a Lyapunov candidate, and consequently the proposed stability and stabilization conditions are wrong.
\end{abstract}

Index Terms - Fuzzy Lyapunov function, Linear matrix inequality (LMI), Takagi-Sugeno (T-S), fuzzy control system, line integral Lyapunov candidate.

\section{INTRODUCTION}

This note considers the work entitled "Fuzzy Control Systems Design via Fuzzy Lyapunov Functions” published by J. Li, S. Zhou and S. Xu in IEEE Trans. on SMC part B [1]. In this paper, the authors propose an extension to the work of Rhee and Won [2]. In the latter, non quadratic stability conditions have been investigated using a line-integral non quadratic Lyapunov Function. This function allows avoiding the membership function derivative in the obtained LMI stability conditions which is the major drawback of classical non quadratic approach. Furthermore, Rhee and Won [2] have proposed an extension to stabilization leading to BMI conditions. In [1], based on a slightly modified line-integral Lyapunov function candidate inspired by [2], the authors intended to provide non quadratic stabilization conditions in the LMI form. Nevertheless, these results have been obtained without taking into account some necessary conditions to ensure the line-integral function to be a Lyapunov function candidate. In this note, it is proved that the proposed stability and stabilization conditions in [1] are wrong. Moreover, it is shown that, to be a non quadratic Lyapunov function candidate, the chosen line-integral function has to fulfill very complex conditions which reduce the interest and applicability of such approach. Finally, an illustrative example is provided that clearly shows that LMI conditions in [1] fail to ensure

Manuscript received February $24^{\text {th }}, 2008$.

Kevin Guelton, Tahar Bouarar and Noureddine Manamanni are with the CReSTIC EA 3804, Université de Reims Champagne-Ardenne, UFR Sciences Exactes et Naturelles, Moulin de la Housse, BP 1039, 51687 Reims cedex 2, France (corresponding author: phone: +33(3)26913261; fax: +33(3)26913106; e-mail: kevin.guelton@univ-reims.fr).

Thierry-Marie Guerra and Miguel Bernal are with the LAMIH UMR CNRS 8530, Université de Valenciennes - Le Mont Houy, F-59313 Valenciennes Cedex 09, France. global asymptotical stable behavior.

\section{II.STATEMENT OF THE PROBLEM}

In [2], a line-integral fuzzy Lyapunov function candidate has been considered such that:

$V(x)=2 \int_{\Gamma(0, x)} f(\psi) \cdot d \psi$

where $\Gamma(0, x)$ is the path from the origin to the current state $x, \psi \in \mathbb{R}^{n}$ is a dummy vector for the integral, $d \psi \in \mathbb{R}^{n}$ is an infinitesimal displacement vector, $f(x) \in \mathbb{R}^{n}$ is a vector function of the state $x$ which can be physically regarded as a force vector. Consequently, (1) represents the work in the force vector field as an energy function.

To be a Lyapunov function candidate, $V(x)$ has to satisfy the following conditions [3]:

1) $V(x)$ is a continuously differentiable function,

2) $V(x)$ is positive definite,

3) $V(x)$ is radially unbounded.

As stated in [2], it is obvious that condition 1) is verified. However, if $V(x)$ is dependent on the path $\Gamma(0, x)$, conditions 2) and 3) would not be satisfied. Hence, it is mandatory to verify the line integral (1) to be pathindependent [4].

Let $f(x)=\left[f_{1}(x), \ldots, f_{n}(x)\right]^{T}$, then the necessary and sufficient condition for $V(x)$ to be path-independent [4] (denoted as lemma 1 in [2]) is given by:

$\frac{\partial f_{i}(x)}{\partial x_{j}}=\frac{\partial f_{j}(x)}{\partial x_{i}}$, for $i, j=1, \ldots, n$.

In [2], a solution satisfying (2) and leading to LMIs (for stability analysis) or BMIs (for stabilization), has been proposed as follows:

$f(x)=\left(\bar{P}+\sum_{i=1}^{r} h_{i}(x) D_{i}\right) x$

with $\quad \bar{P}=\left[\begin{array}{cccc}0 & p_{12} & \cdots & p_{1 n} \\ p_{12} & 0 & \cdots & p_{2 n} \\ \vdots & \vdots & \ddots & \vdots \\ p_{1 n} & p_{2 n} & \cdots & 0\end{array}\right], \quad D_{i}=\left[\begin{array}{cccc}d_{11} & 0 & \cdots & 0 \\ 0 & d_{22} & \ddots & \vdots \\ \vdots & \ddots & \ddots & 0 \\ 0 & \cdots & 0 & d_{n n}\end{array}\right]$,

$\bar{P}+D_{i}=\bar{P}^{T}+D_{i}^{T}>0$ under some special arrangements; see [2] for more details.

Note that, due to condition (2), using a line-integral Lyapunov 
function candidate necessarily leads to a constrained formulation of the vector $f(x)$. In fact, this is the key point of our concern with the work published in [1], since in it the force vector

$$
f(x)=P^{-1}(x) x
$$

with $P(x)=\sum_{i=1}^{r} h_{i}(x) P_{i}$ is used to define the line integral (1).

Then, the authors claim [1]:

"it can be proved that $V(x)$ [i.e. (1) with (4)] satisfies the above conditions 1), 2) and 3). Thus $V(x)$ can be a Lyapunov candidate.”

Unfortunately, this is not always true and the result is not obvious since they didn't take into account the necessary condition (2) for the line-integral to be path-independent. Therefore, theorems in [1] are wrong since $\left\{P_{i}>0\right\}_{i=1}^{r}$ are not properly conditioned by (3).

Naturally, a questions arises: what assumption on $P(x)$ should be done to satisfy (2) with (4)? Let us rewrite:

$$
P^{-1}(x)=\frac{1}{|P(x)|} U(x)
$$

with $U(x)=\left[u_{i j}(x)\right]_{i, j \in\{1, \ldots, n\}}$ being the transpose of the cofactors matrix.

We can write each component of (4) such that for $i=1, \ldots, n$ :

$$
f_{i}(x)=\frac{1}{|P(x)|} \sum_{k=1}^{n} u_{i k}(x) \cdot x_{k}
$$

Thus, it will lead to very complicated relations (if any) to ensure (2). Indeed, from (6), the following equalities should stand for every $x \in \mathbb{R}^{n}$ :

$$
\begin{aligned}
\left(\sum _ { k = 1 } ^ { n } \left(\frac{\partial u_{i k}(x)}{\partial x_{j}}-\right.\right. & \left.\left.\frac{\partial u_{j k}(x)}{\partial x_{i}}\right) \cdot x_{k}+u_{i j}(x)-u_{j i}(x)\right)|P(x)| \\
& +\left(\frac{\partial|P(x)|}{\partial x_{i}}-\frac{\partial|P(x)|}{\partial x_{j}}\right) \sum_{k=1}^{n} u_{i k}(x) \cdot x_{k}=0
\end{aligned}
$$

Let us exhibit this fact on the simplest case. Consider $x=\left[\begin{array}{ll}x_{1} & x_{2}\end{array}\right]^{T} \in \mathbb{R}^{2}$ and $P\left(x_{1}\right)=h_{1}\left(x_{1}\right) P_{1}+h_{2}\left(x_{1}\right) P_{2}$. Since $h_{2}=1-h_{1}$, one can write $P(x)=R+h_{1}\left(x_{1}\right) Q \quad$ with $R=P_{2}=\left[\begin{array}{ll}r_{11} & r_{12} \\ r_{12} & r_{22}\end{array}\right]$ and $Q=P_{1}-P_{2}=\left[\begin{array}{ll}q_{11} & q_{12} \\ q_{12} & q_{22}\end{array}\right]$. It yields:

$$
f_{1}(x)=\frac{1}{\left|P\left(x_{1}\right)\right|}\left(\left(r_{22}+h_{1}\left(x_{1}\right) q_{22}\right) x_{1}-\left(r_{12}+h_{1}\left(x_{1}\right) q_{12}\right) x_{2}\right)
$$

and

$$
f_{2}(x)=\frac{1}{\left|P\left(x_{1}\right)\right|}\left(-\left(r_{12}+h_{1}\left(x_{1}\right) q_{12}\right) x_{1}+\left(r_{11}+h_{1}\left(x_{1}\right) q_{11}\right) x_{2}\right)(9)
$$

Let us denote $|R|=r_{11} r_{22}-r_{12}^{2}, \quad|Q|=q_{11} q_{22}-q_{12}^{2} \quad$ and $S=r_{11} q_{22}+q_{11} r_{22}-2 r_{12} q_{12}$, it is easy to show that: $|P(x)|=h_{1}^{2}\left(x_{1}\right)|Q|+|R|+h_{1}\left(x_{1}\right) S$. Then, from (2) it follows:

$$
\begin{aligned}
& \left|P\left(x_{1}\right)\right| \frac{\partial h_{1}}{\partial x_{1}}\left(q_{11} x_{2}-q_{12} x_{1}\right) \\
& +\frac{\partial h_{1}}{\partial x_{1}}\left(2 h_{1}\left(x_{1}\right)|Q|+S\right)\left(\begin{array}{c}
\left(r_{12}+h_{1}\left(x_{1}\right) q_{12}\right) x_{1} \\
-\left(r_{11}+h_{1}\left(x_{1}\right) q_{11}\right) x_{2}
\end{array}\right)=0
\end{aligned}
$$

or equivalently:

$\frac{\partial h_{1}}{\partial x_{1}}\left(\begin{array}{c}\left(h_{1}^{2}\left(x_{1}\right)|Q|-|R|\right)\left(q_{12} x_{1}-q_{11} x_{2}\right) \\ +\left(2 h_{1}\left(x_{1}\right)|Q|+S\right)\left(r_{12} x_{1}-r_{11} x_{2}\right)\end{array}\right)=0$

which is satisfied for every $x_{1} \in \mathbb{R}$ and $x_{2} \in \mathbb{R}$ if:

$\frac{\partial h_{1}}{\partial x_{1}}\left(\left(h_{1}^{2}\left(x_{1}\right)|Q|-|R|\right) q_{12}+\left(2 h_{1}\left(x_{1}\right)|Q|+S\right) r_{12}\right) x_{1}=0$

and

$\frac{\partial h_{1}}{\partial x_{1}}\left(\left(h_{1}^{2}\left(x_{1}\right)|Q|-|R|\right) q_{11}+\left(2 h_{1}\left(x_{1}\right)|Q|+S\right) r_{11}\right) x_{2}=0$

Note that $r_{11}>0$ because of $R=R^{T}>0$; therefore $|Q|=0$ is mandatory implying $q_{12}= \pm \sqrt{q_{11} q_{22}}$. Then, the conditions to be satisfied are:

$-|R| q_{12}+S r_{12}=0$

and

$-|R| q_{11}+S r_{11}=0$

Several solutions are possible:

1) Recalling that $r_{11}>0$, a first possibility is $S=0$ and consequently $q_{11}=q_{12}=0$ as $|R|>0$. Given that $S=r_{11} q_{22}+q_{11} r_{22}-2 r_{12} q_{12}=0, S=0$ implies $q_{22}=0$ and in conclusion $Q=0$, which resumes to the classical quadratic case. Consequently, this case doesn't help the author's objective of deriving non quadratic stability conditions 
without knowledge on the time-derivative membership function.

2) Another possibility stands for $S \neq 0$ leading to $q_{11} \neq 0$ and $q_{12} \neq 0$. Note that $q_{12}= \pm \sqrt{q_{11} q_{22}}$ also implies $q_{22} \neq 0$. Thus, one can extract $\frac{|R|}{S}=\frac{r_{12}}{q_{12}}=\frac{r_{11}}{q_{11}}$ or equivalently: $r_{12}=r_{11} \frac{q_{12}}{q_{11}}= \pm r_{11} \sqrt{\frac{q_{22}}{q_{11}}}$. Then after some easy manipulations one has $S=q_{11} r_{22}-r_{11} q_{22}$ and $|R|=r_{11} r_{22}-r_{11}^{2} \frac{q_{22}}{q_{11}}$. Therefore, by choosing the following matrices:

$R=\left[\begin{array}{cc}r_{11} & \pm r_{11} \sqrt{\frac{q_{22}}{q_{11}}} \\ \pm r_{11} \sqrt{\frac{q_{22}}{q_{11}}} & r_{22}\end{array}\right], Q=\left[\begin{array}{cc}q_{11} & \pm \sqrt{q_{11} q_{22}} \\ \pm \sqrt{q_{11} q_{22}} & q_{22}\end{array}\right]$,

$P(x)=R+h_{1}\left(x_{1}\right) Q \quad$ is a possible solution for path independence if conditions $R>0$ and $R+Q>0$ are satisfied.

Note that this simple two-rule second-order example leads to complex and constrained conditions for path independence. Consequently, generalizing this result may lead to very complicated and probably unfruitful stability conditions which reduce the interest of such approaches.

\section{ILLUSTRATIVE UNSTABLE EXAMPLE}

Let us consider the following TS model:

$\dot{x}=\sum_{i=1}^{4} h_{i}(x)\left(A_{i} x+B_{i} u\right)$,

where $A_{1}=\left[\begin{array}{cc}1 & -10 \\ 0 & 1\end{array}\right], \quad A_{2}=\left[\begin{array}{cc}0.8 & -11 \\ 0 & 1.3\end{array}\right], \quad A_{3}=\left[\begin{array}{cc}0.9 & -1 \\ 0 & 1.2\end{array}\right]$, $A_{4}=\left[\begin{array}{cc}2 & 0 \\ 10 & 0\end{array}\right], B_{1}=B_{2}=\left[\begin{array}{l}1 \\ 1\end{array}\right], B_{3}=\left[\begin{array}{c}-1 \\ 2\end{array}\right]$ and $B_{4}=\left[\begin{array}{c}-1 \\ 30\end{array}\right]$.

According to the authors, T-S model (16) under control law

$u(t)=\sum_{i=1}^{4} h_{i}(x) N_{i}\left(\sum_{j=1}^{4} h_{j}(x) P_{j}\right)^{-1} x$

is asymptotically stable, with $P_{i}, N_{i}, i=1, \ldots, 4$, satisfying

LMIs in Theorem 4 of [1]. Therefore, since matrices

$$
\begin{aligned}
& P_{1}=\left[\begin{array}{cc}
17.7252 & 8.2080 \\
8.2080 & 6.5760
\end{array}\right], P_{2}=\left[\begin{array}{cc}
19.6434 & 7.8336 \\
7.8336 & 6.3412
\end{array}\right], \\
& P_{3}=\left[\begin{array}{cc}
1.3766 & -2.7913 \\
-2.7913 & 12.4234
\end{array}\right], P_{4}=\left[\begin{array}{cc}
0.0366 & -1.6015 \\
-1.6015 & 197.3652
\end{array}\right], \\
& N_{1}=\left[\begin{array}{ll}
59.0848 & -9.7748
\end{array}\right], N_{2}=\left[\begin{array}{ll}
64.9897 & -11.6187
\end{array}\right],
\end{aligned}
$$

$N_{3}=\left[\begin{array}{ll}6.1237 & -8.1063\end{array}\right], N_{4}=\left[\begin{array}{ll}0.1188 & 0.3410\end{array}\right]$ satisfy LMIs in Theorem 4 of [1], TS model (16) is expected to be globally asymptotically stable under control law (17).

Results in [1] do not define any particular set of membership functions $h_{i}(x)$ for which Theorem 4 can be applied, since they are irrelevant for its conditions. Consider then $w_{1}\left(x_{1}\right)=1-\cos ^{2}\left(x_{1}\right)$ and $w_{2}\left(x_{2}\right)=\frac{e^{\cos \left(x_{2}\right)}}{1+e^{\cos \left(x_{2}\right)}}\left(1-\sin ^{2}\left(x_{2}\right)\right)$ to define membership functions $h_{1}=w_{1} w_{2}, h_{2}=w_{1}\left(1-w_{2}\right)$, $h_{3}=\left(1-w_{1}\right) w_{2}$, and $h_{4}=\left(1-w_{1}\right)\left(1-w_{2}\right)$.

Fig. 1 shows the evolution of the states for TS model (16) under control law (17), from initial conditions $\left(x_{1}, x_{2}\right)=(-5,5),\left(x_{1}, x_{2}\right)=(5,-5)$ and $\left(x_{1}, x_{2}\right)=(-0.5,0.5)$. Although conditions in Theorem 4 of [1] have been fulfilled, the so-called controlled model remains unstable, since the trajectories converge towards a limit cycle. This shows that the proposed conditions failed to ensure any stable behavior.

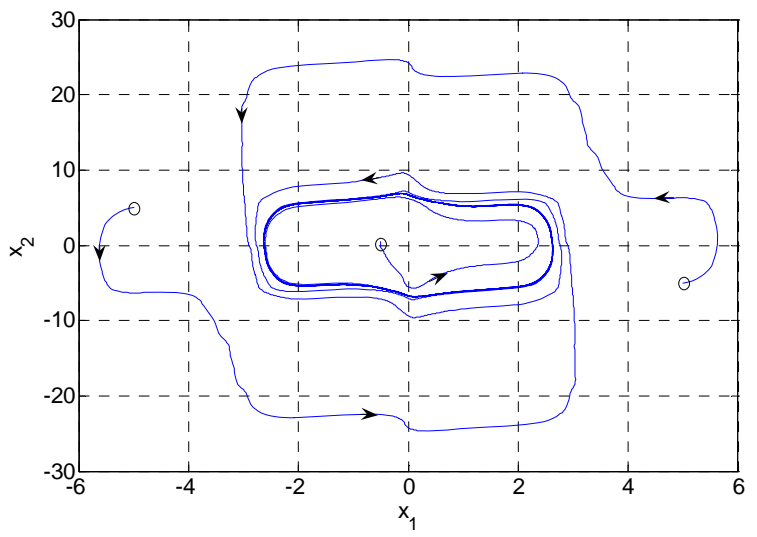

Fig. 1: Evolution of the states of TS model (16) under control law (17).

\section{REFERENCES}

[1] J. Li, S. Zhou and S. Xu, "Fuzzy Control Systems design via Fuzzy Lyapunov Functions", IEEE Transaction on Systems, Man, and Cybernetics - Part B, vol. 38 n 6, pp. 1657-1661, 2008.

[2] B.J. Rhee and S. Won, "A new fuzzy Lyapunov function approach for a Takagi-Sugeno fuzzy control system design”, Fuzzy Sets ans Systems, vol. 157, pp. 1211-1228, 2008.

[3] H.K. Khalil, Nonlinear systems, third ed., Prentice-Hall, Upper Saddle River, NJ, 2002.

[4] D.G. Zill, M.R. Gullen, Advanced Engineering Mathematics, second ed., Jones and Bartlett, London, UK, 2000 